could not be fully obtained as the child's English was limited, and the mother could speak only Portuguese. The father died of unknown cause. Patient always well till the year before, when she had measles; and since then she had not been robust. About a week previous to her entrance to the hospital she began to have pain in the abdomen, paroxysmal in character, and, soon after, swelling of abdomen, vomiting, diarrhoea and headache. On entrance, the diarrhoca had ceased, and there was constipation instead. 'Temperature $104.4^{\circ}$, pulse 135 . Physical examination by Dr. J. J. Minot and myself showed that the patient was a thin, poorly-nourished girl, features pale and pinched. Tongue slightly coated and dry. Heart and lungs negative. Abdomen markedly distended and containing fluid - very sensitive to pressure. An ill-defined mass could be felt in the umbilical region, extending to the right flank. Patient weighed on entrance sixty-five pounds.

July 13 th. Condition much the same as on entrance. Operation performed under ether. An incision was made in the median line about six inches in length. Upon opening peritoneal cavity, it was found filled with serous fluid slightly reddened by blood. Both the parietal and visceral peritoneum were found studded with small, millet-seed nodules; and a little to the right of the umbilicus was found a hard, irregularshaped mass covered with similar nodules, and which on examination proved to be omentum closely matted together and thickened. The intestines, which were held together by soft adhesions, were separated by the hand, and a small piece was snipped off the parietal peritoneum for microscopical examination. The abdominal cavity was then thoroughly flushed out with warm salt-solution, and the wound closed with sterilized Chinese-silk suture, iodoform and baked-gauze dressing. Patient made a good recovery from ether; but towards evening there was considerable shock - the the temperature rising to $104.4^{\circ}$, pulse 150 and weak. Stimulants were freely given by rectum, and about midnight the temperature began to fall a little.

Her condition improved rapidly in the next few days; and in about two weeks she was sitting up and having full diet. Some days later a little pus appeared at one of the stitch-holes; and in a short time every one broke down, making a series of connecting tubercular sinuses. These were thoroughly curetted, under ether, by Dr. Munro, and the cavities packed with iodoform. In two weeks the wound had entirely healed. After this there was a steady gain in strength and weight. Her weight, July 10 th, was 65 pounds; September 12 th, was 68 pounds; October 18 th, was 71 pounds; November $23 \mathrm{th}$, was 86 pounds.

Microscopical examination by Dr. B. F. Mallory of the piece of parietal peritoneum removed at the time of the operation, showed miliary tuberculosis with bacilli. The granulations from the stitch sinuses were examined by Dr. Coggeshall, who also found the tubercle bacilli.

This case seems to be as complete a one of apparent recovery, as can be reported. At every stage we have careful microscopical and bacillary examinations by competent men; we have the weights of the patient and her temperature; and the patient is present tonight to show what the operation of laparotomy can accomplish in a severe case. The great relief following the opening and washing out of the abdomen, the healing of the wound followed by the appearance of a tubercular process creeping up along the tract of the stitches through the abdominal wall, its disappearance after thorough scraping and plenty of iodoform, and the presence now of the patient in perfect health, make it complete.

One point very strongly emphasized here is that drainage should never be used unless it is of absolute necessity on account of the pressure of pus or a very large amount of cheesy detritus, neither of these conditions being found, as a rule, in cases of general tubercular peritonitis. The benefit or harm done by drainage in this class of tubercular disease is determined by the same, or nearly the same, rules and conditions as in other parts of the body. Personally, I most thoroughly believe in closing without drainage every cavity resulting from an operation on tubercular processes not already suppurating. This rule applies not only to serous cavities but also to other sites of the disease. If through faulty asepsis or other reasons pus torms, it is then time enough to drain by gauze or tube. I am not prepared to say whether the introduction of iodoform into a wound to be closed by suture is of benefit or not. My results with and without it are about equal.

König states that the cases in his list show that those washed out with antiseptics show the best percentage of results. I am not ready to introduce into the abdominal cavity any known antiseptic in sufficient quantity or of sufficient strength to produce any action on the micro-organisms present, especially as so many cases have been cured without their use.

In conclusion, I quote the words of Osler whose article must, in the main, stand as the most useful one that we have on the subject:

"The important practical point, however, is the relief and cure of these cases by laparotomy; and the surgeons may well leave to the pathologist the minor question of determining the nature - whether tibroid, lymphomatous, or tubercular - of the chronic peritonitis."

\section{Elinical \$Department.}

\section{A CASE OF PLACEN'TA PREVIA lateralis.}

BY HUBER' G. WILBUR, M.D., FALL RIVKR, MA89.

Mrs. V., age thirty-five, of good personal and family history, the mother of four children, when about three months preguant began flowing at irregular intervals. The hamorrhage was slight at first and painless. Later on it was more abundant, so that she felt weak, and became alarmed. On March 13th, just a few days before $I$ saw her, the flowing was unusually profuse. She was then apparently about six months along. The foetal heart was heard. Vaginal examination was negative.

A consultation was held with Dr. Gifford; and in view of the recent severe hremorrhage, operation was decided upon. The expectant method did not seem wise, since the parents did not care especially to save the child.

Ether was given, and the vagina cleansed by a douche, 1-5000 bichloride; then with aseptic precautions the os was dilated manually. Version was performed, and the fœetus extracted rapidly. The placenta was felt in the left side just about at the margin of the inner cervical ring. 
The hamorrhage during and after the operation was considerable. 'The uterus did not contract well, presumably from the ether. I think there was some collcealed hamorrhage, for the patient was blanched, the pulse small and weak; and the flowing for two or three hours after the operation was very profuse. I was unable to give ergot at once owing to the partially unconscious condition. Stimulants were given freely, however, subcutaneously ; and a large enema of salt solution was introduced, so that gradually the patient revived.

'Ten hours later she was seized with severe pain in the left side, which was controlled fully by a subcutaneous injection of morphia. Subsequently, for two or three days, there was a little temperature, which gradually disappreared. Hot vaginal douches (1-3000 bichloride) were given twice daily after the operation until the temperature subsided. With supportive treatment and abundant nourishment, the patient is now entirely well.

\section{ghedical Brogregg:}

\section{RECEN'I PROGRESS IN SURGERY.}

HY H. L. BURHELL, M.D., AND H. W. CUSHING, M.1).

PENTAL AS AN ANASTHETIC.

Thene are collected 226 cases where pental was used for anasthesia, and there has already been a fatal case recorded. Schede ${ }^{1}$ advises that its use should be restricted.

LOCAL ANASTHESIA BY DILUTE SOLUTIONS.

In order to avoid danger from the toxic effects of cocaine as ordinarily used, Schleich, ${ }^{2}$ of Berlin, endeavored to learn by persoual experimentation the weakest solution of cocaine that was effective. 'This was a one-fiftieth-per-cent. solution. He found that distilled water injected subcutaneously was able to cause local anæethesia, thus confirming the observations of Dawbarn, of New York. 'The water caused pain during injection, which was not noticed when a twoper-cent. salt solution was used. When tho salt solution was combined with cocaine, a solution in distilled water of two-per-cent. salt and one-hundredth-percent. cocaine was found to give local anasthesia enough for practical purposes. The advantage of this solution is that a large amount can be used, and without danger of toxic effect; also large areas can be uffected.

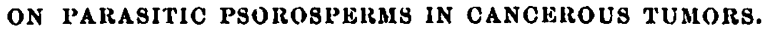

Ruffer ${ }^{8}$ states that since his first note on this subject, in conjunction with J. Herbert Walker, they have been able further to confirm the opinion expressed at that time. He describes the intranuclear stage of the parasite. 'They are usually found in groups of two or three, and sometimes as high as twenty. When filled with spores the nucleus appears as a hard, dark-brown mass with irregular outlines. The psorosperm first appears in the nucleus in the cancer-cell. $\mathrm{As}_{8}$ it dovelops, it becomes more trausparent, and each parasite has in it a small nucleus. They are found most commonly in the extremely soft cancer of the breast.

1 Is Somalno Mćdicale, 1882, No. 31.

2 Annals of Surgory, 1802 vol. xvi, 163 .
PYOKTANIN FOR THE CURE OF CANCEL.

$A$ recent review of the literature on this subject, by Idelson, ${ }^{4}$ of Berne, seems to indicate that the value or efficiency of pyoktanin in the cure of epithelioma is not yet established, and that the successes are out numbered by failures.

\section{ARE BULLETS STERILE?}

This question which has so frequently been answered in the affirmative has been investigated by Messner, of Wiesbaten, and his conclusions are that the heating of the bullet during the discharge of the gun is not enough to destroy any bacteria which may be adherent to it nor even to impede their development. His experiments were made by shooting a bullet into a tin can filled with sterilized Koch meat peptonegelatine. A full charge of powder was exploded. The range was 125 and 250 metres. Tho conditions were as follows: (1) A non-infected bullet, can sterile. (2) Bullet infected (pure culture), can sterile. (3) Bullet non-infected, can sterile, but covered with a flannel covering which was infected with a known coccus, pure culture.

'The results are given thus :

(1) The shot canal through the gelatine, showed no change or more frequently a more or less vigorous growth of "scheinmelpilz," or those varieties wore commonly found in the air.

(2) Without exception did the gelatine contained in the can slow the development of the specific germ with which the bullet was infected.

(3) Here also the shot canal was infected with the same germ with which the flannel covering of the can was infected.

Although the experiments in the third class are not wholly convincing, still enough data is furnished by the rest of the work to decide the question in the negative.

\section{CEREBRAL, TUMORS.}

At the German Surgical Congress, 'Twenty-first Session, 1892, Bramann, of Halle, reports two successful cases of removal of cerebral tumors. One was a small, round-cell sarcoma, in a man forty-six years old, on whom three operations were performed. 'The other was also a man, age twenty-six; tumor weighed nine ounces.

In the resulting discussion it was found that the frequency of cerebral tumors was one in eighty-four autopsies (Seydel, Munich), and one in fifty-nine (Hale White, London). 'The latter's records include more children; hence more tubercular tumors and the difference. Seydel's list of one hundred cases showed 27 tuberculous, 39 sarcomas (gliosarcomata and gliomata), 2 cysticerci, 1 actinomycosis, 3 cholesteatoma, 2 cysts, 2 carcinomata, 2 psammommata, 6 syphilitic, 16 not specified. 'The discussion with reference to the percentage of cases suitable for operation, that is, where the tumor was not too large, not metastatic, not multiple and was situated on the surface of the brain, is of interest. The conclusions were that the percentage of such cases is quite small. Seydel found three in one hundred; Hale White nine, of which v. Bergmann rejected seven, reducing the number to two; Starr sixteen in three thousand. 'There are at present on record fivo hundred cases of brain tumors (postmortem statistics), of which eleven (2.2 per cent.)

Annals of Surgery, 1802, vol. x7l, 88.

" Muchu. mod. Wohnsohr., 1802, xxxix, 23. 